\title{
SÔBRE O USO DO EXTRATO GLICERINADO DE HIPÓFISES DE PEIXE NA REPRODUÇÃO DOS PEIXES DOS AÇUDES DO NORDESTE DO BRASIL
}

Rui Simões de Menezes - Osmar Fontenelle - Emilio C. Camacho

Comissão Técnica de Piscicultura (IFOCS, MVOP)

- Fortaleza, Ceará, Brasil. -

0 uso de um extrato glicerinado de hipófises de peixe para obter a reprodução dos peixes existentes no Brasil foi aplicado, pela primeira vez, em São Paulo, por Fonseca Ribeiro e TABARElli Neto (6). R. S. MEnEzes usou-o, com relativo sucesso, durante a piracema de 1943-1944, na Estação Experimental de Caça e Pesca, Pirassununga, S. Paulo, às margens do Rio Mogí-Guaçú (4) .

No Nordeste brasileiro, durante as piracemas (quadras de reprodução dos peixes) de 1944 (março a maio) e de 1945 (janeiro a março), tivemos oportunidade de usar diversas partidas de extrato glicerinado, com o objetivo de metodizar sua aplicação para os peixes regionais.

\section{1 - MATERIAL E MÉTODOS}

O extrato glicerinado foi preparado no Laboratorio do Pôsto de Piscicultura de Fortaleza, rigorosamente de acôrdo com a técnica descrita por Fonseca Ribeiro e TABARElli Neto (6). Diversas partidas dêsse extrato foram preparadas, como se verifica pela Tabela I.

$\mathrm{O}$ número de peixes reprodutores hipofisados com extrato glicerinado, nos Postos de Piscicultura de Fortaleza e Lima Campos (Ceará), atingiu ao total de 219 , assim distribuido:

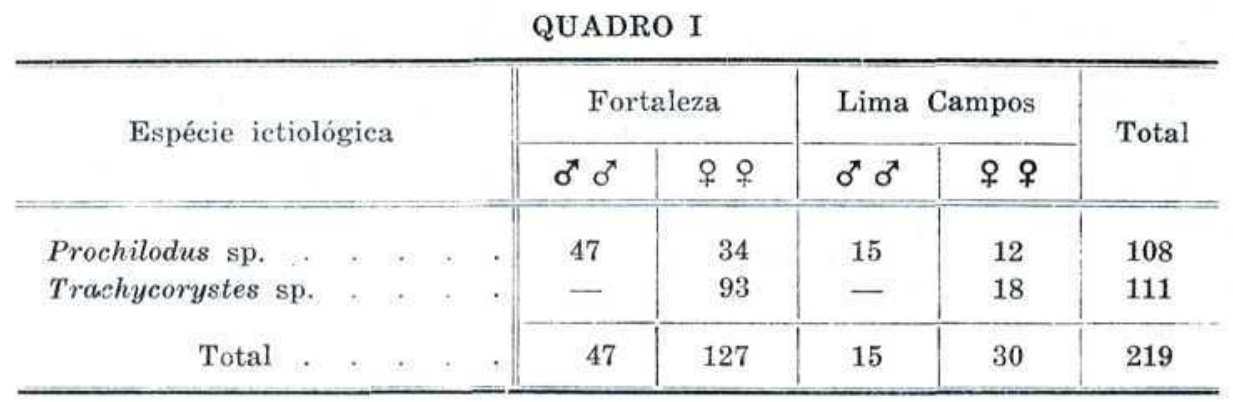

Estão condensados nas Tabelas II e III os principais detalhes das hipofisações efetuadas em Fortaleza e Lima Campos, respectivamente. 
O metódo das hipofisações foi o mesmo anteriormente descrito (4 e 6).

\section{2 - DISCUSSÃO}

Teremos, inicialmente, de discutir os resultados obtidos em cada um dos Postos de Piscicultura - Fortaleza e Lima Campos.

1 - Fortaleza: - Analisemos, separadamente, os resultados obtidos com ambas as espécies ictiológicas hipofisadas.

a) Prochilodus sp.: - A posologia e intervalos entre as doses, adotados para os lotes A, C, D, E, F, G, em 1944, podem ser considerados desfavoráveis, (o intervalo, para o lote G, é uma exceção). Demonstra-o uma rápida análise percentual dos resultados obtidos.

\begin{tabular}{c|c|c|c|c}
\multicolumn{5}{c}{ QUADRO II } \\
\hline \multirow{2}{*}{ Lotes } & Poreentagem & Positividade Reprodutores & \multicolumn{2}{c}{ Positividade Lotes } \\
\cline { 2 - 5 } & $\sigma^{\pi} \sigma$ & $\% \%$ & Pareial & Total \\
\hline \hline A & $75 \%$ & $100 \%$ & + & - \\
C & $50 \%$ & - & - & - \\
D & - & - & - & - \\
E & $100 \%$ & $66,6 \%$ & + & - \\
F & $50 \%$ & - & - & - \\
G & $100 \%$ & $25 \%$ & + & - \\
\hline
\end{tabular}

OBSERVAÇÃO: - Considerado positivo o lote em que houve positividade nos reprodutorıł masculinos e femininos: positivo parcial quando a positividade não foi de $100 \%$ em ambos os sexos; positivo total, quando de $100 \%$ em ambos os sexos.

A análise percentual dos lotes K (1944) e D, E, F (1945), de posologia e intervalos diferentes, revela o seguinte:

\begin{tabular}{l|c|c|c|c}
\multicolumn{6}{c}{ QUADRO III } \\
\hline \multirow{2}{*}{ Lotes } & Porentagem Positividade Reprodutores & \multicolumn{2}{|c}{ Positividade Lotes } \\
\cline { 2 - 5 } & $\delta \%$ & $q \%$ & Parcial & Total \\
\hline K (1944) & $100 \%$ & $100 \%$ & - & + \\
D (1945) & $100 \%$ & $100 \%$ & - & + \\
E (1945) & $100 \%$ & $66,6 \%$ & + & - \\
F (1945) & $100 \%$ & $66,6 \%$ & + & - \\
\hline
\end{tabular}

OBSERVACÃO: - Considerado positivo o lote em que houve positividade nos reprodutore masculinos e femininos; positivo parcial, quando a positividade nấo foi de $100 \%$ em ambos os sexos; positivo total. quando de $100 \%$ em ambos os sexos. 
R. S. de Menezes, O. Fontenele e E. C. Camacho - Reprodução dos peixes 177

O estudo comparativo dos quadros II e III evidencia que:

$\left.1 .^{\circ}\right)$ - no quadro II, a porcentagem de $100 \%$ de positividade dos reprodutores masculinos, dentro dos lotes respectivos, foi de $33,2 \%$;

$2 .^{\circ}$ ) - no quadro III, a porcentagem de $100 \%$ de positividade dos reprodutores masculinos, dentro dos lotes respectivos, foi de $100 \%$;

$3 .^{\circ}$ ) - no quadro II, a porcentagem de $100 \%$ de positividade dos reprodutores femininos, dentro dos lotes respectivos, foi de $16,6 \%$;

$4 .^{\circ}$ ) - no quadro III, a porcentagem de $100 \%$ de positividade dos reprodutores femininos, dentro dos lotes respectivos, foi de $50 \%$;

$5 .^{\circ}$ ) - no quadro II não houve nem um lote com positividade total ;

$6 .^{\circ}$ ) - no quadro III, $50 \%$ dos lotes tiveram positividade total.

b) Trachycorystes sp.: - O pequeno número de lotes experimentados, bem como as características especiais da reprodução desta espécie e, consequentemente, a sua hipofisação (hipofisados sòmente os reprodutores femininos), levaram-nos a considerar positivos apenas os lotes "em que houve desova com evolução completa do ôvo" (1).

Resume o quadro abaixo os principais detalhes técnicos concomitantes e decorrentes da hipofisação dos lotes A, B (1944), B, C, (1945):

QUADRO IV

\begin{tabular}{|c|c|c|c|c|c|c|}
\hline \multirow{2}{*}{ Anos } & \multirow{2}{*}{ Lotes } & \multirow{2}{*}{ 우오 } & \multirow{2}{*}{ Proveniência } & \multirow{2}{*}{$\begin{array}{l}\text { Epoca das } \\
\text { hipofisaçốes }\end{array}$} & \multicolumn{2}{|c|}{ Produção ovos (1.) } \\
\hline & & & & & Total & "Per Capita" \\
\hline 1944 & A & 41 & Aç. Columinjuba & 17 a $18-4$ & 5,350 & 0,130 \\
\hline 1944 & B & 29 & $"$ & 21 a $22-4$ & 5,400 & 0,186 \\
\hline 1945 & B & 15 & $"$ & 7 a $8-3$ & 3,000 & 0,200 \\
\hline 1945 & $\mathrm{C}$ & 8 & $\begin{array}{l}\text { Açs. Columinjuba } \\
\text { e Brisamar }\end{array}$ & 12 a $13-3$ & 1,100 & 0,137 \\
\hline
\end{tabular}

Da produtividade "per capita", visto a proveniência dos reprodutores e proximidade da época de hipofisação, verifica-se que as doses aplicadas nos lotes B (1944), B e C (1945) foram mais eficazes.

2 - Lime Campos: - Analisemos, separadamente, os resultados obtidos com ambas as espécies ictiológicas hipofisadas; embora a massa de dados experimentais desta localidade seja bem inferior à de Fortaleza (45 reprodutores, em 5 lotes, para Lima Campos; 174 reprodutores, em 14 lotes, para Fortaleza). 
a) Prochilodus sp.: - A posologia adotada para os lotes $\mathrm{F}$ e G, em 1944, pode ser considerada desfavoravel, visto terem sido negativos os seus resultados (Tab. III). A posologia adotada para o lote $\mathrm{H}$, mesmo dadas suas condições peculiares de re-hipofisação, pode ser considerada favoravel porque:

1. $\left.^{\circ}\right)$ - o resultado foi positivo;

$2^{\circ}$ ) - a posologia que condicionou êsse resultado positivo foi aplicada, com idêntico sucesso, nos lotes K (1944), D, E, F (1945), do Pôsto de Piscicultura de Fortaleza, conforme se verifica pela Tabela II e Quadro III.

b) Trachycorystes sp.: - A pequena massa de dados experimentais, consistindo em dois lotes hipofisados simultâneamente, para fins especiais de verificação de potencialidade do hormônio gonadotropico do extrato (partidas 2 e 3, Tabela I), nos leva a abandonar qualquer discussão sôbre posologia e intervalos entre as doses. Cingimo-nos, para esta espécie, apenas aos dados experimentais colhidos em Fortaleza (Quadro IV).

\section{RESUMO}

Após várias pesquisas sôbre o uso do extrato glicerinado de hipófises de peixe na reprodução dos peixes dos açudes do Nordeste do Brasil, os autores concluem que:

$1^{\circ}$ ) - a posologia do extrato deve ser: $-0,25 ; 0,50 ; 1,00$; 1,$50 ; 3,00$ hipófises.

$2 .^{\circ}$ ) - esta posologia comporta, tambem, a seguinte variante: - 0,$25 ; 0,50 ; 1,00 ; 2,00 ; 4,00$ hipófises.

$3 .^{\circ}$ ) - há maior uniformidade no teor de hormônio gonadotropico do extrato, pela maior quantidade de hipófises utilizadas.

$\left.4 .^{\circ}\right)$ - é evitado o uso de hipófises conservadas em álcool absoluto, que dá menor uniformidade no teor de hormônio gonadotropico, pela menor quantidade de hipófises utilizadas.

5. ${ }^{\circ}$ ) - é indicado fixar um intervalo de 8 horas entre as doses sucessivas.

\section{SUMMARY}

After several researches upon the use of glycerin extract of hypophysis from donor fishes in the breeding of Fishes obtained from Northeast Brasil dams, the Authors concludes that:

1 st - the extract posology should be: - 0,25; 0,50;1,00; 1,$50 ; 3,00$ hypophysis.

2nd - the extract posology also should be: - 0,25; 0,50; 1,$00 ; 2,00 ; 4,00$ hypophysis. 
$3 r d$ - a greater uniformity in the gonadotropic hormone content in extract it is due to the greater amount of utilized hypophysis.

4th - the use of preserved hypophysis in absolute alcohol it is avoided, which gives a lesser uniformity in the gonadotropic hormone content, as consequence of lesser amount utilized hypophysis. 5th - a eight hours interval between the successive doses is indicated.

\section{BIBLIOGRAFIA}

1 - Azeveido, P. DE - Oliveira, A. C. Estevão DE - 1939 - Sôbre o emprêgo da hipófise conservada em álcool absoluto na desova dos peixes. "in" Livro de homenagem aos Profs. Álvaro e Miguel Ozório de Almeida, 35-42

2 - Fontenele, O. - Camacho, E. C. - Menezes, R. Simões de - 1946 Obtenção de três desovas anuais de Curimatã, Prochilodus sp., (PISCES: CHARACIDAE, PROCHILODINAE), pelo método de hipofisação. (Nota prévia.) "Bol. Mus. Nacional, Zool.", N. S. (53) : 1-9

3 - Menezes, R. SimôEs DE - 1943 - O método de hipofisação de peixes na piscicultura. O Campo, Rio de Janeiro, $14(160)$ : 39-44. Segunda edição, revista e aumentada, publicada em Julho, 1945, mimeografada, em Fortaleza, constituindo a Publicação n. ${ }^{\circ} 102$ da Comissão Téenica de Piscicultura

4 - Menezes, R. Simões DE -1944 - Nota sôbre a hipofisação de peixes do rio Mogí-Guaçú com extrato glicerinado de hipófises de peixe. Bol. Ind. Anim., S. Paulo, N. S., 7 (3/4) : $36-44$

5 - Menezes, R. Simões DE - 1945 - Ação de hipófises de peixes doadores em diestro sôbre peixes reprodutores em estro. Rev. Brasil. Biol., 5 (4): 535-9

6 - Fonseca Ribeiro - Tabarelli Neto, J. F. - 1944 - Da obtenção de um extrato glicerinado para a hipofisação dos peixes. Rev. Fac. Med. Vet., S. Paulo, 2 (4) : 227-32. 
TABELA 1

Características das partidas de extrato glicerinado de hipófises de peixe

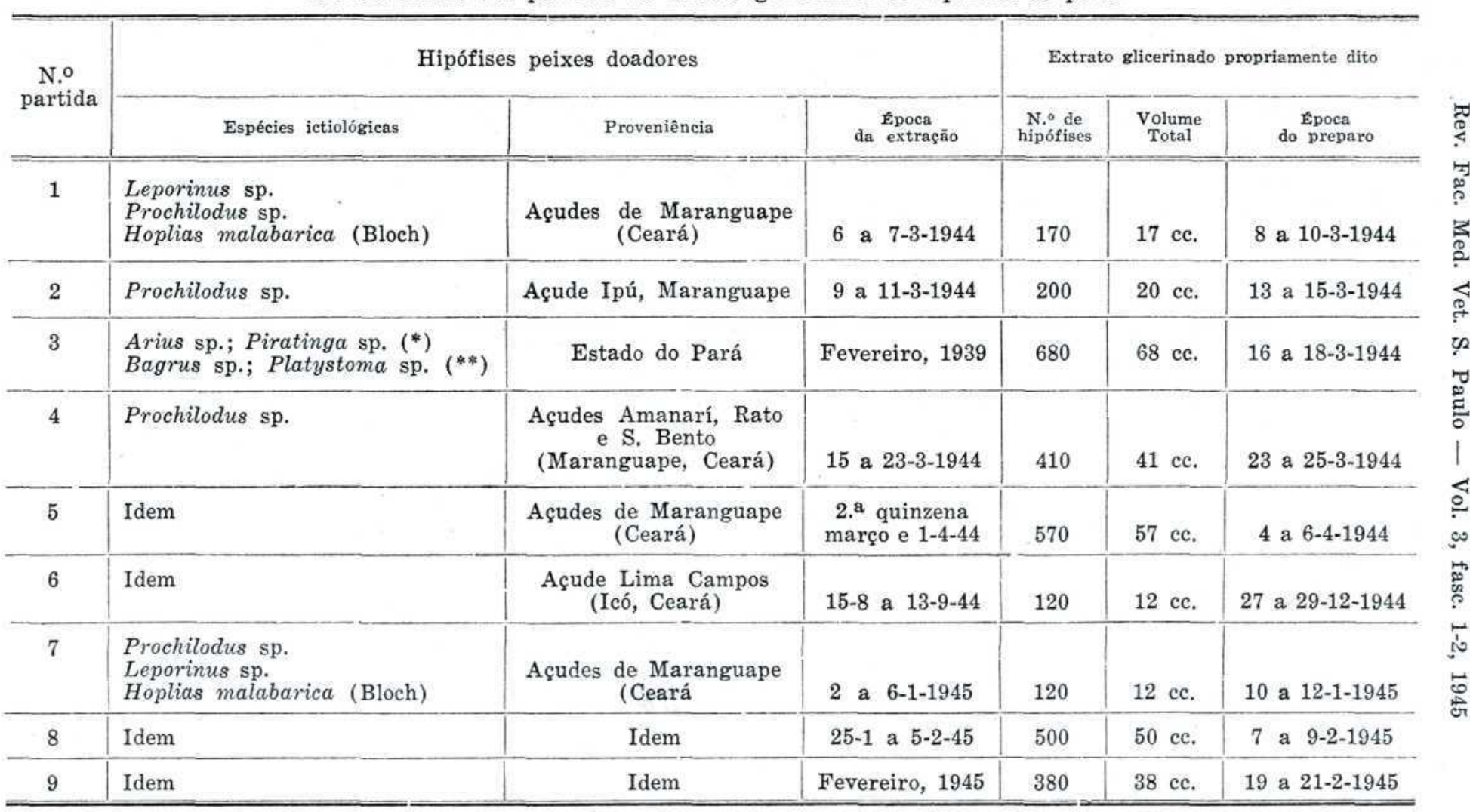

(*) Caiu em sinonimia de Brachyplatystoma
(*) Nome pré-ocupado. 
TABELA 2

Hipofisações em Fortaleza (Ceará)

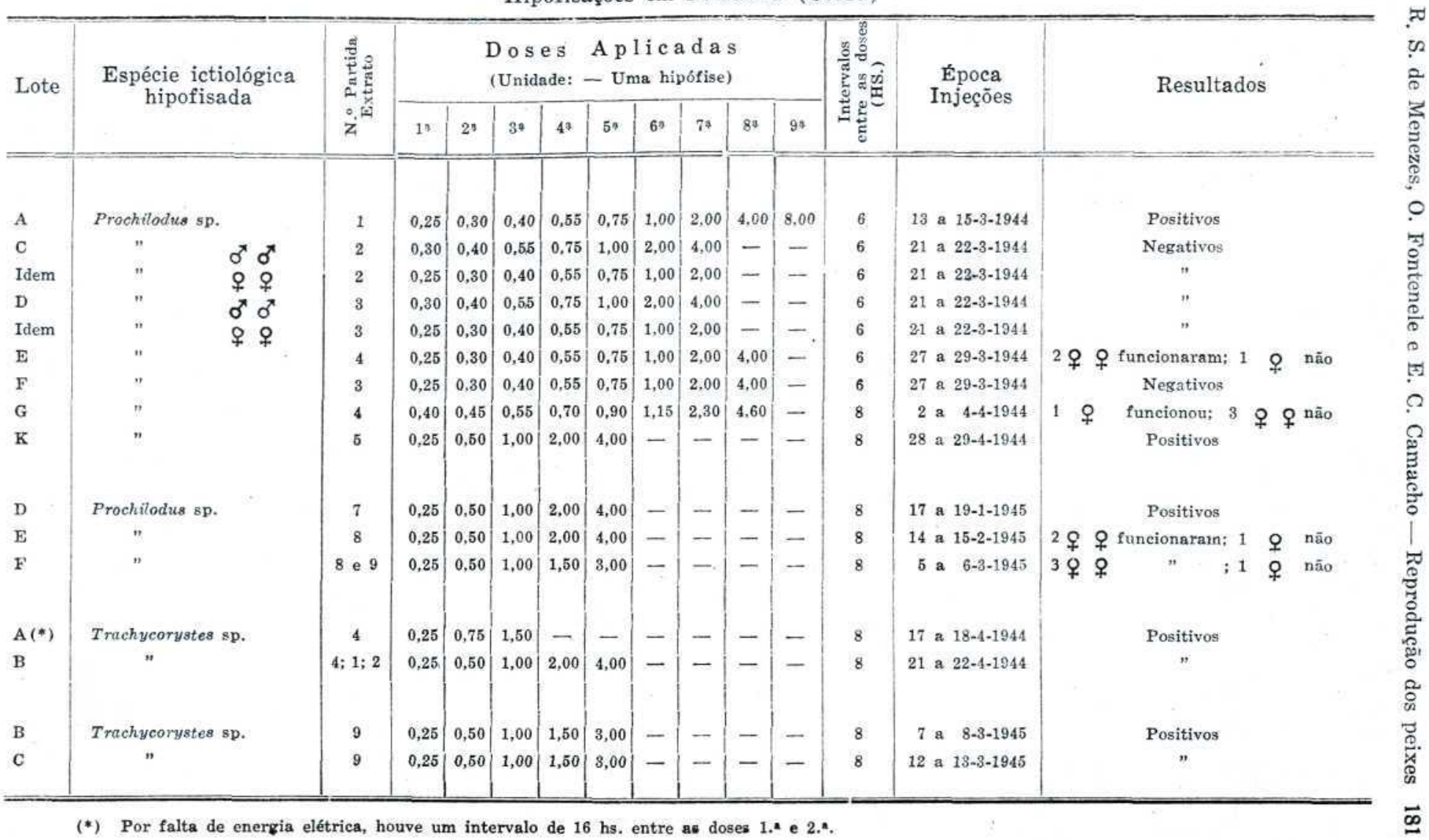


TABELA 3

Hipofisações em Lima Campos (Icó, Ceará)

\begin{tabular}{|c|c|c|c|c|c|c|c|c|c|c|c|c|}
\hline \multirow{2}{*}{ Lote } & \multirow{2}{*}{$\begin{array}{c}\text { Espécie ictiológica } \\
\text { hipofisada }\end{array}$} & \multirow{2}{*}{ 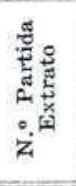 } & \multicolumn{7}{|c|}{$\begin{array}{l}\text { Doses Aplicadas } \\
\text { (Unidade: - Uma hipófise) }\end{array}$} & 焉 & \multirow{2}{*}{$\begin{array}{l}\text { Época } \\
\text { Injeções }\end{array}$} & \multirow[t]{2}{*}{ Resultados } \\
\hline & & & $1 .{ }^{\mathrm{a}}$ & $2,{ }^{a}$ & $3 . \mathrm{a}$ & $4 .^{2}$ & $5 .{ }^{*}$ & $6 \cdot^{n}$ & $7 . \overline{1}$ & 范 & & \\
\hline $\mathrm{F}$ & Prochilodus sp. $\left(\sigma^{\star} \sigma^{\pi}\right)$ & 2 & 0,15 & 0,25 & 0,40 & 0,70 & 1,00 & - & - & 6 & 2 a $3-4-1944$ & Negativos \\
\hline Idem & $" \quad(q q)$ & 2 & 0,20 & 0,30 & 0,45 & 0,75 & 1,00 & - & - & 6 & $"$ & $"$ \\
\hline G & $" \quad\left(\alpha^{x} \alpha^{x}\right)$ & 3 & 0,15 & 0,25 & 0,40 & 0,75 & 1,00 & - & - & 6 & $"$ & $"$ \\
\hline Idem & $" \quad(\% \circ)$ & 3 & 0,20 & 0,30 & 0,45 & 0,75 & 1,00 & - & - & 6 & $"$ & $"$ \\
\hline $\mathrm{H}(*)$ & $"$ & 5 & 0,25 & 0,50 & 1,00 & 2,00 & - & - & - & 6 & 24 a $25-5-1944$ & Positivos \\
\hline B & Trachycorystes $\mathrm{sp}$. & 2 & 0,30 & 0,50 & 0,75 & 1,00 & 1,50 & - & - & 8 & 22 a $23-3-1944$ & Positivos \\
\hline C & $"$ & 3 & 0,30 & 0,50 & 0,75 & 1,00 & 1,50 & 2,00 & 2,00 & 8 & $"$ & Negativos \\
\hline
\end{tabular}

(*) Material hipofisado anteriormente, com resultados positivos, com hipófises conservadas em áiencl absoluto. 